\title{
Novel mutations associated with autosomal-dominant congenital cataract identified in Chinese families
}

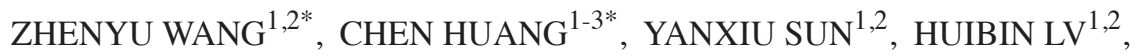 \\ MINGZHOU ZHANG ${ }^{1,2}$ and XUEMIN LI ${ }^{1,2}$ \\ ${ }^{1}$ Department of Ophthalmology; ${ }^{2}$ Beijing Key Laboratory of Restoration of Damaged Ocular Nerve; \\ ${ }^{3}$ Medical Research Center, Peking University Third Hospital, Beijing 100191, P.R. China
}

Received October 25, 2018; Accepted July 5, 2019

DOI: $10.3892 / \mathrm{etm} .2019 .7865$

\begin{abstract}
As the leading cause of impaired vision, congenital cataracts, particularly autosomal dominant congenital cataract (ADCC), have been considered as a hereditary disease. The present study aimed to identify genetic defects in Chinese pedigrees with ADCC. A total of 6 Chinese families with ADCC were included, comprising 103 members and 27 patients assessed in total. Genomic DNA samples were extracted from the peripheral blood of probands; mutations were determined using a specific eye disease enrichment panel with next-generation sequencing. Following pathogenicity prediction, sites with notable pathogenicity were screened for further validation. Sanger sequencing was performed in the remaining individuals of the families and 100 normal controls. The pathogenic effects of the mutations, including amino acid substitutions, as well as structural and functional alterations of proteins linked to ADCC, were investigated via bioinformatics analysis. A total of seven mutations in six candidate genes associated with ADCC were identified in the 6 families: Myosin heavy chain 9 (MYH9) c. $4150 \mathrm{G}>\mathrm{C}$, $\beta$-crystallin A4 (CRYBA4) c.169T >C, RPGR-interacting protein 1 (RPGRRIP1) c.2669G $>$ A, wolframin (WFS1) c.1235T >C, CRYBA4 c.26C $>$ T, Ephrin receptor subfamily 2 (EPHA2) c.2663+1G $>$ A and paired box 6 (PAX6) c.11-2A $>$ G. The seven mutations were only detected in affected individuals. Among them, there were three novel mutations (MYH9: c.4150G>C; CRYBA4: c.169T>C; RPGRRIP1: c.2669G>A) and four previously reported ones. Mutations in RPGRIP1 (c.2669G $>$ A) and CRYBA4 (c.26C $>$ T) were predicted to be benign according to bioinformatics analysis. Conversely, other mutations in EPHA2, PAX6, MYH9, CRYBA4 (c.169T>C)
\end{abstract}

Correspondence to: Dr Xuemin Li, Department of Ophthalmology, Peking University Third Hospital, 49 North Garden Road, Haidian, Beijing 100191, P.R. China

E-mail: 13911254862@163.com

*Contributed equally

Key words: cataract, genomic, myosin heavy chain $9, \beta$-crystallin 4 and WFS1 were determined to be pathogenic. The present study reported two novel heterozygous mutations (MYH9 c.4150G $>$ C and CRYBA4 c.169T>C) identified by analyzing 6 Chinese families with ADCC, supporting their important roles in the development of the disease.

\section{Introduction}

During the initial stages of vision development, lens opacity may arise, which is mainly caused by congenital cataracts and may lead to deprivation amblyopia (1). According to the literature, approximately one-third of infantile blindness cases are caused by congenital cataracts $(2,3)$. The incidence of congenital cataracts is $6.31 / 100,000$ individuals (4) in industrialized countries, while that in developing countries is assumed to be notably higher $(5,6)$.

As the worldwide leading cause of impaired vision in children, the hereditary modes of congenital cataracts include autosomal dominant, autosomal recessive and X-linked hereditary modes. Among these modes, autosomal-dominant congenital cataract (ADCC) is most common (4); variable phenotypes occur in different families $(7,8)$. To date, $\geq 23$ genes have been associated with ADCC. These genes are mainly involved in the formation of the lens, including the crystallin (CRY) genes $[\alpha$-CRY (CRYA), $\beta$-CRY (CRYB) and $\gamma$-CRY (CRYG)], lens-specific connexin (Cx) genes (Cx43, Cx46 and $\mathrm{Cx} 50$ ), major intrinsic protein gene or aquaporine, cytoskeletal structural protein genes, paired-like homeodomain transcription factor 3 , avian musculoaponeurotic fibrosarcoma, heat shock transcription factor 4 , beaded filament structural protein 2 and non-muscle myosin heavy chain IIA (MYH9) $(7,9,10)$. In addition, ephrin receptor subfamily (EPHA)1 and -2, RPGR-interacting protein 1 (RPGRIP1) and paired box 6 (PAX6) serve a vital role in the pathogenesis of cataracts.

Of note, mutations in the same gene may lead to different phenotypes $(7,8)$. For individuals in the same family, ADCC may present with different clinical features. The present study reported variable clinical features in patients from the same family as well as from different families. Therefore, in the present study, targeted gene capture was performed using a hereditary-eye-disease-enriching panel and next-generation sequencing to identify the mutations of six Chinese families with ADCC, including two novel mutations in MYH9 
(c.4150G>C) and CRYBA4; c.169T>C). The results of the present study may provide insight into the mutations associated with the development of ADCC.

\section{Subjects and methods}

Recruitment of patients and clinical evaluation. A total of 6 Chinese families with 103 members in total $(96$ members alive) were recruited from the Peking University Third Hospital (Beijing, China). The pedigree charts are provided in Fig. 1. The probands were as follows: II-1 in family 1; IV-7 in family 2 ; IV-1 in family 3; III-4 in family 4; III-2 in family 5 and III-2 in family 6 . A total of 27 patients were affected by ADCC ( 4 patients from family $1 ; 6$ patients from family 2 ; 4 patients from family $3 ; 7$ patients from family $4 ; 3$ patients from family 5 and 3 patients from family 6). Detailed family and medical histories, and a series of results from ophthalmic examinations, were obtained for the family members, including visual acuity, slit lamp examination and fundus examination with dilated pupils. A total of 100 normal controls were also recruited. All participating individuals provided informed consent in accordance with the Declaration of Helsinki. The present study was approved by the Peking University Third Hospital Medical Ethics Committee (Beijing, China).

Genomic DNA extraction. Venous blood ( $2 \mathrm{ml})$ was collected from the participating family members and was stored in BD Vacutainers (BD Biosciences) containing EDTA to prevent coagulation. Genomic DNA was extracted from the white blood cells using a DNA Extraction kit (Tiangen Biotech Co., Ltd.), and was quantified with a NanoDrop 2000 spectrophotometer (Thermo Fisher Scientific, Inc.).

Mutation screening. Following the extraction of DNA from the white blood cells of the probands from in each family, a specific eye disease enrichment panel was used to capture the gene mutations in the samples (cat. no. OT021-29; MyGenostics GenCap Enrichment Technologies, Inc.). A minimum of $3 \mu \mathrm{g}$ DNA was used for analysis in the indexed Illumina libraries according to the manufacturer's protocols (MyGenostics GenCap Enrichment Technologies, Inc.). The target genes in the enriched libraries were captured in accordance with the MyGenostics Targeted Genes Capture protocols and were then sequenced on an Illumina NextSeq 500 sequencer (Illumina, Inc.) for paired-end reads of $150 \mathrm{bp}$.

A total of 663 disease-associated genes in the panel were linked to hereditary eye diseases. Among these genes, 135 were associated with cataracts (57 genes were associated with congenital cataracts; the others were associated with hereditary eye diseases with opacified lens).

Following sequencing, raw image files were processed using Bcl2Fastq software (Bcl2Fastq 2.18.0.12; Illumina, Inc.) for base calling and raw data generation. Low-quality variations were filtered out using a quality score $\geq 20$. Short Oligonucleotide Analysis Package (SOAP) aligner software (SOAP2.21; soap.genomics.org.cn/soapsnp.html) was then used to align the clean reads to the reference human genome. PCR duplicates were removed and single nucleotide polymorphisms (SNPs) were identified by the GATK (version 4.1.2.0; http://www.broadinstitute.org/gsa/wiki/index.
php/Home_Page) and the SOAPsnp (http://soap.genomics. org.cn/soapsnp.html) programs. Identified SNPs and insertion/deletions were annotated using the Exome-assistant program (http://122.228.158.106/exomeassistant).

DNA samples from other individuals of the families were used to validate all mutations identified by Sanger sequencing on an ABI3730XL analyzer (Applied Biosystems; Thermo Fisher Scientific, Inc.). The coding regions of the candidate genes [MYH9, CRYBA4 (c.169T>C), RPGRRIP1, wolframin (WFS1), CRYBA4 (c.26C>T), EPHA2 and PAX6] were amplified by PCR: An initial denaturation of $98^{\circ} \mathrm{C}$ for $30 \mathrm{sec}, 15$ cycles of denaturation at $98^{\circ} \mathrm{C}$ for $25 \mathrm{sec}$, annealing at $65^{\circ} \mathrm{C}$ for $30 \mathrm{sec}$, extension at $72^{\circ} \mathrm{C}$ for $30 \mathrm{sec}$, and a final extension of $72^{\circ} \mathrm{C}$ for $5 \mathrm{~min}$; primers are listed in Table I. The coding regions were then screened by using bidirectional sequencing followed by analysis with Chromas 2.33 (http://technelysium. com.au/wp/chromas/); comparisons were made using reference sequences in the National Center for Biotechnology Information (NCBI) database.

Bioinformatics analysis. Based on the results obtained from the mutation analysis, several bioinformatics analyses were performed. The potential effects of an amino acid substitution on the structure and function of a protein were predicted using Protein Variation Effect Analyzer (PROVEAN v1.1.3; http://provean.jcvi.org/index.php) (11) Sorting intolerant from tolerant (SIFT; http://sift.bii.a-star.edu.sg/) (12), Mutation Taster (http://www.mutationtaster.org) (13), Polymorphism and Phenotyping version 2 (PolyPhen-2; http://genetics.bwh. harvard.edu/pph2/) (14) and Swiss model (https://swissmodel. expasy.org) (15).

\section{Results}

Clinical features. Analysis of the family history and medical history indicated that none of the patients had any other systemic diseases that may be associated with the development of cataracts or ophthalmic diseases. In the present study, 27 patients with ADCC ( 25 alive) in 6 Chinese families were identified, including 9 males and 18 females (16 alive). Their slit lamp examination images are presented in Fig. 2. Among these patients, opacified lens was observed at a young age in certain subjects, including patients in families 2, 4, 5 and 6 . The youngest patient of the 6 families was 3 months old. The parents observed an opacified lens for the first time when the patient was 2 months old. The non-transparent area quickly progressed in the past 2 months and eventually, the whole lens was opacified (Fig. 2G); patients in families 1 and 3 gradually developed symptoms of ADCC after 11 years of age.

Of note, the proband of family 1 and their mother (suffering from ADCC) presented with a dissimilar clinical feature that affected her visual acuity in a different manner (Table II). The proband of family 1 was a 13-year-old female with irregular nuclear cataracts in the bilateral eyes (Fig. 2A) and her visual acuity was 20/200 OU. However, her mother with the same mutation in the MYH9 gene only presented with mild symptoms of ADCC; spot-like opacity was observed in the peripheral area of the lens (Fig. 2B), which resulted in a slight reduction in visual acuity (20/25 OU). 
Family 1

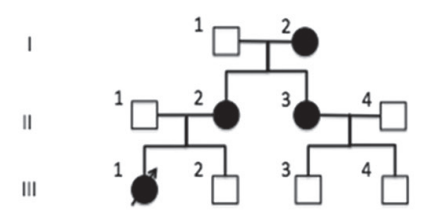

Family 2

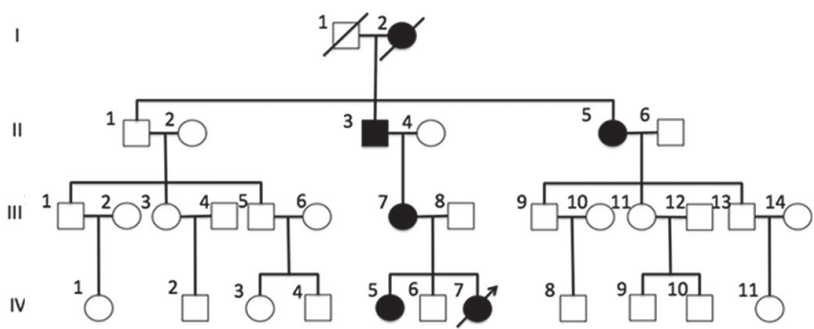

Family 3

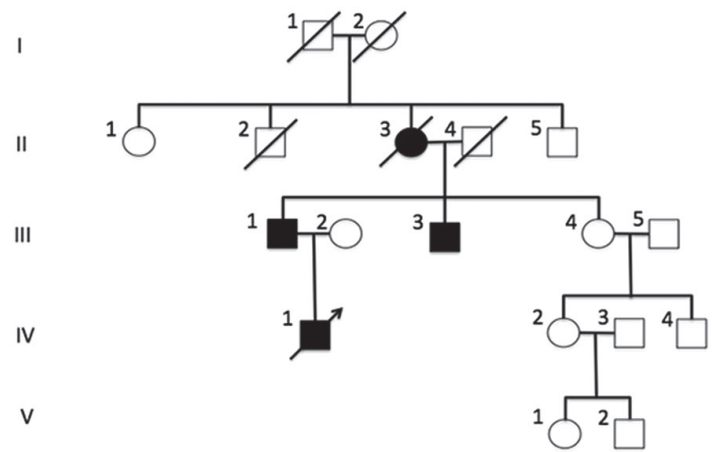

Family 4

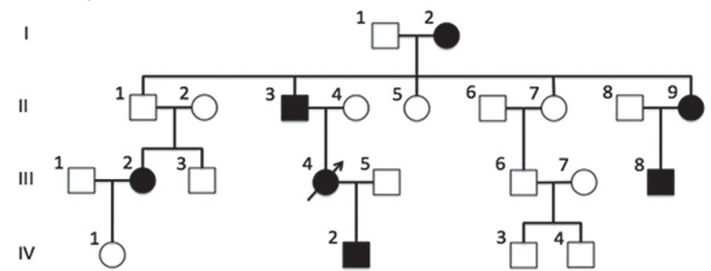

Family 5

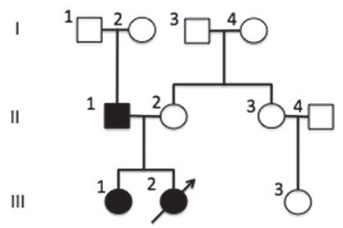

Family 6

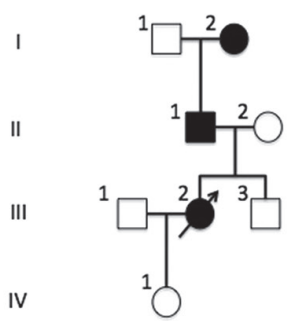

Figure 1. Pedigree of six families with autosomal-dominant congenital cataracts. Squares and circles indicate males and females, respectively, and crossed symbols indicate deceased subjects. Black symbols indicate affected individuals and the proband of each family is indicated by an arrow.

Mutation screening and bioinformatics analysis. High-throughput screening of the blood samples of all of the probands was performed in the present study. Compared with the normal gene sequences, each proband had $>3,000$ nucleotide alterations in hereditary eye disease-associated genes. Few of these nucleotide alterations did not result in changes in the amino acid sequence or were not in accordance with the hereditary model of ADCC; thus, these alterations were excluded. Only the genes known to be associated with ADCC were selected. Among them, variants in MYH9, RPGRRIP1, WFS1, EPHA2 and PAX6 of the probands were indicated to be potentially pathogenic. Sanger sequencing was performed with the blood samples of other individuals in the families. The results revealed that heterozygous MYH9 c.4150G >C, CRYBA4 c.169T>C, RPGRIP1 c. $2669 \mathrm{G}>\mathrm{A}$, WFS1 c.1235T $>$ C, CRYBA4 c. $26 \mathrm{C}>\mathrm{T}, \mathrm{EPHA} 2$ c. $2663+1 \mathrm{G}>\mathrm{A}$ and PAX6 c. $11-2 \mathrm{~A}>\mathrm{G}$ mutations were only detected in affected individuals. These mutations were not detected in the 100 controls and were considered to be associated with ADCC (Fig. 3). The evolutionary conservation results of these genes were also shown in Fig. 4.

A novel damaging missense mutation: MYH9 c.4150G $>C$. The mutation c.4150G $>C$ in MYH9 was identified in family 1 (Fig. 3A). This missense mutation led to an amino acid substitution from glutamate to glutamine at codon 1,384 (p.E1384Q) in MYH9.

According to the analysis with Mutation Taster, glutamate was highly conserved at codon 1,384 of MYH9 among different species (Fig. 4A). Substituting glutamine at this codon was predicted to be pathogenic by Mutation Taster ('disease-causing'), PROVEAN ('deleterious' with a score of -2.64), SIFT ('damaging' with a score of 0.023) and PolyPhen-2 ('probably damaging' with a score of 1.000 , sensitivity of 0.00 and specificity of 1.00).

A novel damaging missense mutation: CRYBA4 c.169T $>C$. The c.169T $>$ C mutation in CRYBA4 was identified in family 4 (Fig. 3B). This missense mutation led to an amino acid substitution from phenylalanine to leucine at codon 57 (p.F57L) in CRYBA4.

According to analysis with Mutation Taster, phenylalanine is conserved at codon 57 of CRYBA4 in different species (Fig. 4B). This mutation was predicted to be pathogenic by Mutation Taster ('disease-causing'), PROVEAN ('deleterious' with a score of -5.08), SIFT ('damaging' with a score of 0.002) and PolyPhen-2 ('probably damaging' with a score of 0.968 , sensitivity of 0.74 and specificity of 0.96 ). The three-dimensional (3D) models of wild-type and mutated CRYBA4-encoding protein were then generated by Swiss model (Fig. 5). 


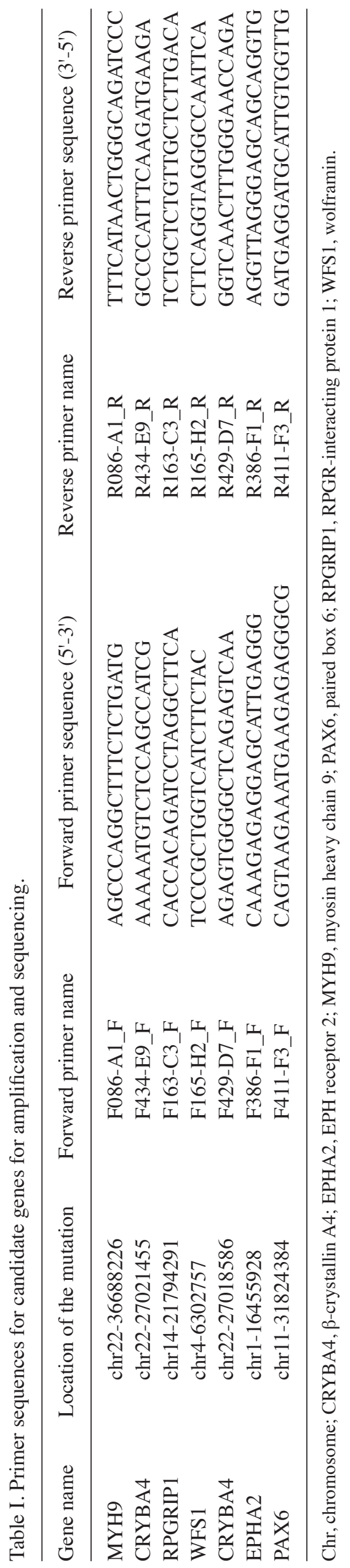

A novel neutral missense mutation: RPGRIP1 c.2669G $>A$. The c.2669G $>$ A mutation in RPGRIP1 was identified in family 2 (Fig. 3C). This missense mutation may cause a 'Neutral' (PROVEAN prediction with a score of 1.41) and 'Tolerated' (SIFT prediction with a score of 0.867) amino acid substitution from arginine to glutamine at codon 890 (p.R890Q) in RPGRIP1. The arginine residue was not highly conserved in different species (Fig. 4C). The prediction made with Mutation Taster was 'polymorphism' and PolyPhen-2 analysis scored this mutation as 0.000 (sensitivity: 1.00; specificity: 0.00).

A previously reported missense mutation: WFS1 c.1235T $>C$. The mutation c.1235T $>\mathrm{C}$ in WFS1 was identified in family 5 (Fig. 3D). This missense mutation leads to an amino acid substitution from valine to alanine at codon 412 (p.V412A).

Analysis with Mutation Taster revealed that valine is conserved at codon 412 of WFS1 in different species (Fig. 4D); the mutation was then predicted to be pathogenic by Mutation Taster ('disease-causing'), SIFT ('damaging' with a score of 0.021 ) and PolyPhen-2 ('probably damaging' with a score of 0.981 , sensitivity of 0.75 and specificity of 0.96 ). However, analysis with PROVEAN indicated a prediction of 'neutral' with a score of -2.29 .

This mutation was first reported by Choi et al (16) as a candidate gene for familial nonsyndromic hearing loss; however, according to the results for family 5 , it may be possible that this mutation also causes ADCC.

A reported missense mutation: CRYBA4 c.26C $>T$. The c. $26 \mathrm{C}>\mathrm{T}$ mutation was identified in CRYBA4 in family 6 (Fig. 3E), which causes an amino acid substitution from alanine to valine at codon 9 (p.A9V). This mutation has been reported by Sun et al (17) and Zhai et al (18).

The present study reported the clinical features of ADCC in the families analyzed; however, bioinformatics analysis indicated that this mutation may not be pathogenic. According to the results of bioinformatics analysis, it is possible that the c. $26 \mathrm{C}>\mathrm{T}$ mutation is not responsible for the disease. According to Mutation Taster, alanine is conserved in different species (Fig. 4E). On the contrary, the mutation was then predicted to cause a 'polymorphism' using Mutation Taster. In addition, mutation of the amino acid (p.V412A) was predicted by PROVEAN, SIFT and PolyPhen-2. Prediction with PROVEAN suggested that this mutation is 'neutral' with a score of -0.27 . SIFT indicated that the mutation is a 'tolerated' mutation with a score of 0.502 . The prediction with PolyPhen-2 was 'benign' and this mutation was scored as 0.009 (sensitivity: 0.96; specificity: 0.77 ).

Zhai et al (18) proposed co-segregation of this mutation in ADCC; however, further investigation is required.

A reported splicing mutation: EPHA2 c.2663+1G $>A$. Following mutation screening, a splice donor site mutation was determined in EPHA2 (c.2663+1G >A, chr-16455928; Fig. 3F) in family 2 . The screening results revealed that the mutation is located in the first region of Intron 16 and it was predicted to induce a substitution from aspartic acid to glutamate. In addition, it was predicted to lead to the formation of a stop codon, causing the loss of 34 amino acids encoded by exon 17 (19). 
Table II. Clinical features of selected patients.

\begin{tabular}{|c|c|c|c|c|c|}
\hline $\begin{array}{l}\text { Family } \\
\text { number }\end{array}$ & $\begin{array}{c}\text { Affected } \\
\text { individual }\end{array}$ & Gender & Age & Visual acuity & Phenotype \\
\hline 1 & III-1 & Female & $13 \mathrm{y}$ & $20 / 200$ & Nuclear cataract \\
\hline 1 & II-2 & Female & $35 \mathrm{y}$ & $20 / 25$ & Spot-like cataract in the peripheral area of the lens \\
\hline 2 & IV-7 & Female & $3 \mathrm{~m}$ & Not determined & Irregular spot-like cataract in the middle of the lens \\
\hline 3 & IV-1 & Male & $14 \mathrm{y}$ & $20 / 40$ & Two round-shaped opacifications in the middle of the lens \\
\hline 4 & III-4 & Female & $22 \mathrm{y}$ & $20 / 200$ & Irregular nuclear cataract \\
\hline 4 & II-3 & Male & $53 \mathrm{y}$ & $20 / 200$ & Irregular nuclear cataract \\
\hline 5 & III-2 & Female & $4 \mathrm{~m}$ & Not determined & Extensive opacification of the lens \\
\hline 6 & III-2 & Female & $23 \mathrm{y}$ & $20 / 100$ & Round-shaped opacification in the middle of the lens \\
\hline
\end{tabular}

$\mathrm{y}$, years; m, months.
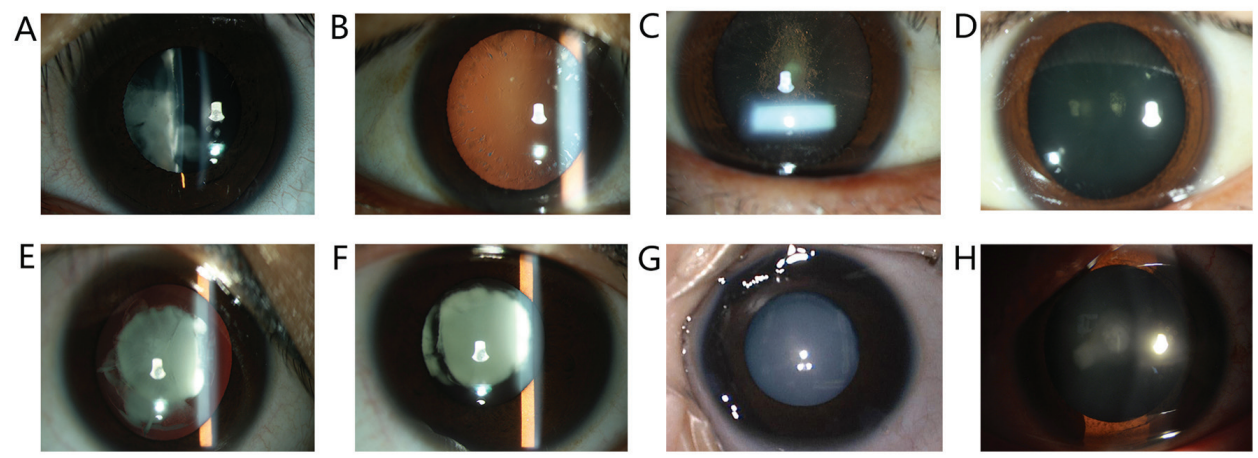

Figure 2. Slit lamp images of the eyes of various family members. (A) In the proband of family 1 (III-1), irregular opacities in the lens were observed. (B) Subject no. II-2 in family 1, the mother of the proband. (C) Proband of family 2 (IV-7). (D) Proband of family 3 (IV-1). (E) Proband of family 4 (III-4). (F) Subject no. II-3 in family 4, the father of the proband. (G) Proband of family 5 (III-2). (H) Proband of family 6 (III-2).

It was not possible to predict the severe consequences of this mutation using SIFT, PROVEAN and PolyPhen2; however, the degree of damage associated with this mutation was investigated using Mutation Taster and GERP++ prediction tools. The mutation in EPHA2 received a GERP score of 5.77 and was considered to be a conserved site. Mutation Taster analysis indicated disease-causing effects, as sequence motif loss and loss of protein were reported.

This mutation has been previously reported by Bu et al (19); the mutation in EPHA2 is located in chromosome 1-16455928. It is possible that EPHA2 is able to exhibit a variety of mutation types. The present study reported the mutation in EPHA2 as c. $2663+1 \mathrm{G}>\mathrm{A}$ using the NCBI database.

A reported splicing mutation: PAX6 c.11-2A $>G$. The mutation in PAX6 was also identified in family 3 as a splicing mutation in intron 4 , which is located in two regions prior to the beginning of exon 5. This mutation was predicted to lead to a substitution of serine (encoded by TCA) to arginine (encoded by TCG). It was predicted to result in the addition of 56 amino acids (Fig. 3G) (20).

SIFT, PROVEAN and PolyPhen2 analyses were not able to make any prediction regarding the mutation in PAX6; however, according to the GERP++ score of 4.9 and Mutation Taster score of 1 , the site was determined to be highly conserved among different species. Mutation Taster predicted the mutation to be 'disease-causing'. From the 3D models of wild-type and mutated PAX6, the additional amino acids were observed to form an abnormally long 'tail', which may affect the function of PAX6 (Fig. 5). This mutation was also reported by Churchill et al (20).

\section{Discussion}

Congenital cataracts are the leading cause of impaired vision in pediatric patients worldwide (1). According to recent studies, $\sim 45 \%$ of families with a history of cataracts have been reported to carry mutations in the CRY genes; $\sim 12 \%$ have mutations in the genes encoding various growth or transcription factors. In addition, $16 \%$ have mutations in Cx-encoding genes; 5\% possess mutations in intermediate filament proteins, membrane proteins or protein degradation-associated genes. Furthermore, $\sim 8 \%$ have mutations in a variety of other functionally diverse genes. Inheritance of the same mutation in different families or in different individuals within the same family may result in distinct cataract phenotypes (phenotypic heterogeneity). This suggests that additional genetic or environmental factors may affect the expression of the mutant protein, which may be the primary cause of cataracts $(7,8)$. On the contrary, mutations in different genes involved in biological processes that are 


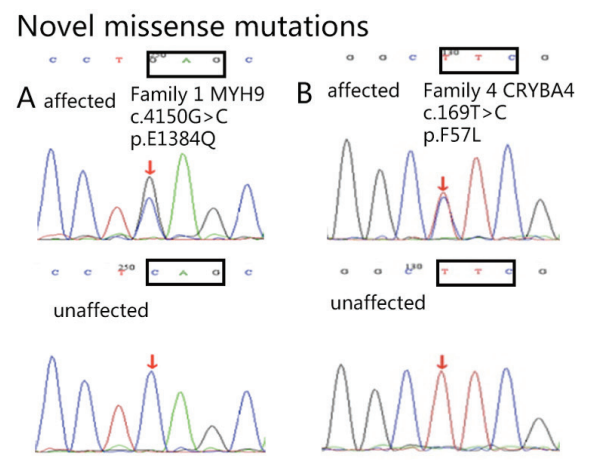

Reported splicing mutation

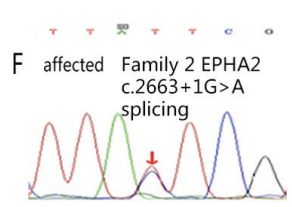

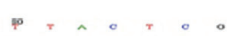

unaffected

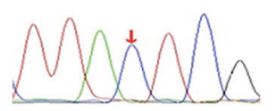

T.

$G$ affected Family 3 PAX6 c. $11-2 A>C$

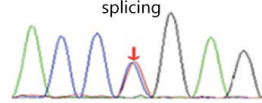

^ ${ }^{-} \circ \times$ so

unaffected

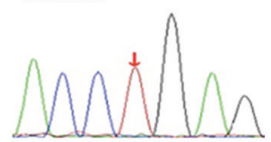

Reported missense mutations
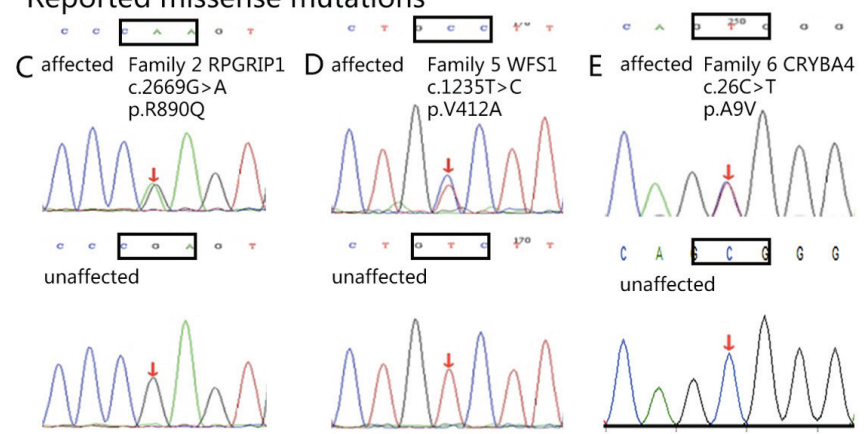

\section{c. $2663+1$ \\ Exon 16 \\ Intron 16 \\ Exon 17 stop codon}

WT mRNA CGUGUC.....CAACGAGUAGU......CCACAGCGACAU......AUCUGA......

Mut mRNA GUGUC......CAACGAAUAAGU......CCACAG CGACAU......AUCUGA...

New stop condon

c. $11-2 A>G$

Exon 4

Intron 4

WT mRNA .....AUGCAGAACAGUAA......AUGAGG......CUCAGGUCACAGCGGA

Mut mRNA .....AUGCAGAACA GUAA......AUGAGGA......CUCGGGUCACAGCGGA....

Figure 3. DNA sequences of affected and unaffected individuals. (A) MYH9 (c.4150G>C). (B) CRYBA4 (c.169T>C). (C) RPGRIP1 (c.2669G>A). (D) WFS1 (c.1235T>C). (E) CRYBA4 (c.26C>T). (F) EPHA2 (c.2663+1G>A); the splicing mutation was determined in the first loci of intron 16, which leads to the substitution from aspartic acid (D, encoded by GAC) to glutamate (E, encoded by GAA), as well as the generation of a new stop codon. This new stop codon causes the loss of 34 amino acids encoded by exon 17. (G) PAX6 (c.11-2A>G); the c.11-2A>G mutation in the PAX6 gene leads to the substitution from serine (S, encoded by AGU) to arginine (R, encoded by AGA) and the addition of 56 amino acids. Associated codons are indicated with rectangles. CRYBA4, $\beta$-crystallin A4; EPHA2, EPH receptor 2; MYH9, myosin heavy chain 9; PAX6, paired box 6; RPGR-interacting protein 1; WFS1, wolframin; WT, wild-type; Mut, mutant.

potentially possibly not linked with each other may lead to cataracts with similar or identical morphologies (genotypic heterogeneity). This suggests that cataract may be the ultimate common pathway for different spectra of initial insults (21). For example, CRYBA4 belongs to crystallin family. It is part of a gene cluster with beta-B1 (CRYBB1), beta-B2 (CRYBB2) and beta-B3 (CRYBB3). Any mutation of these genes may affect the synthesis of crystallins, resulting in opacification of lens (22).

In the present study, a specific panel that included 135 cataract-associated target genes was used to perform screening tests on 6 Chinese families with congenital cataracts. A total of 7 suspected mutations were detected in six genes, including two novel deleterious missense mutations (MYH9 c.4150G $>$ C and CRYBA4 c.169T $>$ C). Amongst them, MYH9 c.4150G $>$ C, CRYBA4 c.169T $>$ C, RPGRRIP1 c.2669G >A, WFS1 c.1235T>C and CRYBA4 c. $26 \mathrm{C}>\mathrm{T}$ were determined to lead to amino acid changes.
EPHA2 c. $2663+1 \mathrm{G}>\mathrm{A}$ and PAX6 c. $11-2 \mathrm{~A}>\mathrm{G}$ were denoted as splicing mutations.

The c. $4150 \mathrm{G}>\mathrm{C}$ variant is a missense mutation in exon 31 of MYH9 and leads to the substitution of glutamate to glutamine at codon 1,384 (p.E1384Q) in MYH9. In addition, this mutation was not detected in 100 normal controls, suggesting that it is a pathogenic mutation rather than a polymorphism. Bioinformatics analyses indicated that this mutation is a disease-causing mutation. Thus, these results suggest that congenital cataracts in family 1 may have been caused by the mutation in exon 31 of MYH9.

Mutations in MYH9 may cause MYH9-associated disorders, which are characterized by congenital macrothrombocytopenia, accompanied with young-adult-onset sensorineural hearing loss, nephropathy and congenital cataracts; however, the etiologies of these diseases remain elusive (23). To date, several studies have investigated mutations in MYH9 (23-33; Table III). According to Pecci et al (34), cataracts did not 


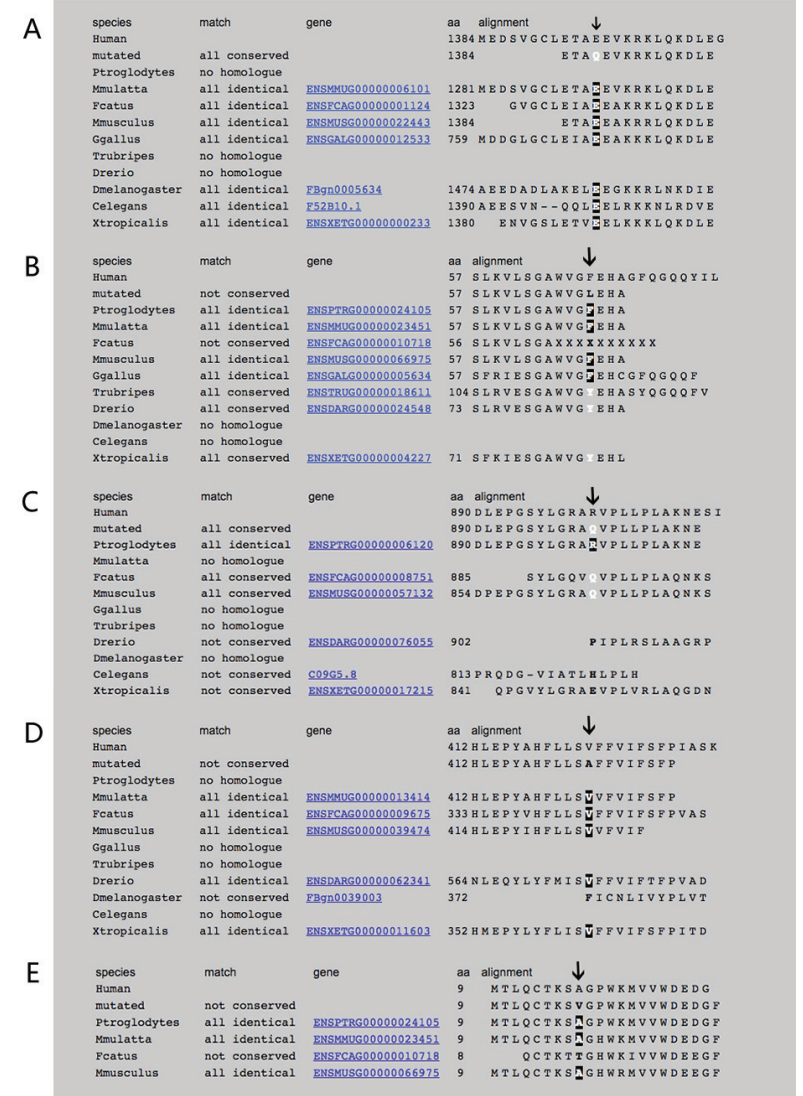

Figure 4. (A) Glutamate E at position 1,384 of myosin heavy chain 9 is highly conserved in different species (indicated by an arrow). (B) Phenylalanine at position 57 of CRYBA4 is conserved in different species (indicated by an arrow). (C) Arginine R at position 890 of RPGR interacting protein 1 is not highly conserved in the majority of species (indicated by an arrow). (D) Valine $\mathrm{V}$ at position 412 of wolframin is conserved in different species (indicated by an arrow). (E) Alanine A at position 9 of CRYBA4 is conserved in different species (indicated by an arrow). CRYBA4, $\beta$-crystallin A4.

commonly occur in a family with a MYH9-associated disorder and were only observed in 43 of 235 patients (18\%); among them, there were four congenital forms of disease. In the present study, family 1 had the ocular manifestation of congenital cataracts without renal complications and hearing loss; the symptoms of these patients were notably mild.

MYH9, mapped to chromosome 22q12.3, includes 47 exons, which encode MYH9 protein. The transcription of this gene begins with the first ATG codon of the open reading frame in exon 2 , which terminates at the stop codon in exon 41 . The mutation identified in the present study was located in exon 31 , which may affect the tail domain of MYH9. According to Pecci et al (34), there was a strong correlation between the genotype and clinical features of patients with MYH9-associated disorders. In addition, mutations in the rod-tail domain have been associated with a mild phenotype (35). Thus, it may be proposed that mutations in exon 31 may lead to hereditary disease, but with a notably mild phenotype, as observed by the manifestations in family 1 . Furthermore, this mutation was not detected in 100 normal controls, suggesting that it is a pathogenic mutation rather than a polymorphism. Bioinformatics analysis suggested that this mutation was a disease-causing mutation. Thus, these results support that congenital cataracts in family 1 may be caused by a mutation in exon 31 of MYH9.
The missense mutation (c.169 T>C) in CRYBA4 was reported to lead to an amino acid substitution from phenylalanine to leucine at codon 57 (p.F57L) in CRYBA4. This mutation was not detected in 100 normal controls and was predicted to be pathogenic by various bioinformatics analyses.

CRYBA4 belongs to the CRYB family of proteins. The CRYB family (35\%), CRYA (40\%) and CRYG are major members of the CRY protein family detected in the mammalian lens (36). CRY proteins are markedly stable in the lens for the maintenance of transparency and refractive ability. CRYB comprises 7 protein forms, each of which is encoded by six genes (CRYBA1, CRYBA2 and CRYBA4, as well as CRYBB1, CRYBB2 and CRYBB3). Among them, CRYBA4 constitutes 196 amino acids and pathogenic mutations have been reported in exons 2 and $4(18,37-39)$ (Table IV). According to Billingsley et al (36) and Zhou et al (40), the mutation in exon 4 serves an important role in the onset of congenital cataracts. The novel mutation detected in the present study is also in this region.

The 3D models of wild-type and mutated CRYBA4 revealed the substitution of phenylalanine at codon 57 with leucine. The mutation was determined to be located in a $\beta$-sheet, which is stabilized by hydrogen bonds. The substitution from phenylalanine to leucine may break these hydrogen bonds, reducing the stability of the secondary structure of the CRYBA4 protein.

In the present study, a c.1235T $>\mathrm{C}$ mutation was identified in WFS1 in family 5 . This missense mutation may cause an amino acid substitution from valine to alanine at codon 412 (p.V412A). The mutation was predicted to be pathogenic by Mutation Taster, SIFT and PolyPhen-2; however, PROVEAN suggested that the mutation was 'neutral' with a score of -2.29.

The WFS1 gene encodes a transmembrane protein expressed by brain, pancreatic, heart and insulinoma $\beta$ cell lines. Mutations in this gene may cause Wolfram syndrome, which leads to numerous conditions, including diabetes insipidus, diabetes mellitus, optic atrophy and deafness. This mutation was first reported by Choi et al (16) as a candidate gene for familial nonsyndromic hearing loss; however, the present study reported the mutation to be associated with the development of ADCC.

Considering the differences between splicing mutations, and those that lead to amino acid substitutions and alterations in protein structure, various bioinformatics tools were employed to determine the consequences of these mutations. The present study reported that, compared with the amino acid substitution mutations, splicing mutations may be more dangerous, as gene expression may be affected by newly formed stop codons or the addition of amino acids. These alterations may cause the loss of motifs, or changes in the structure and function of proteins. The results of the present study revealed that the mutation EPHA2 c. $2663+1 \mathrm{G}>\mathrm{A}$ created a new stop codon, which resulted in the loss of 34 amino acids encoded by exon 17. The mutation PAX6 c.11-2A>G was predicted to cause the addition of 56 amino acids. Further investigation is required to determine the mechanisms underlying the pathological effects of these mutations.

The methods applied to detect the mutations in the present study are of high significance. Considering the number of individuals in the families and the number of cataract-associated 
Table III. Mutations in myosin heavy chain 9 associated with congenital cataracts.

\begin{tabular}{|c|c|c|c|c|}
\hline Amino acid changes & Mutation type & Family origin & Pattern of inheritance & (Refs.) \\
\hline p.E1066_A1072del & Deletion & Japanese & $\mathrm{AD}$ & Aoki T et al (23) \\
\hline p.E1066_A1072del & Deletion & Italian & $\mathrm{AD}$ & Seri et al (24) \\
\hline p.E1066_A1072del & Deletion & Japanese & $\mathrm{AD}$ & Miyazaki et al (25) \\
\hline p.Q1068_L1074 del & Deletion & French & $\mathrm{AD}$ & Saposnik et al (26) \\
\hline p.E1066_A1072dup & Duplication & Italian & $\mathrm{AD}$ & De Rocco D et al (27) \\
\hline p.D1424N & Substitution & Japanese & $\mathrm{AD}$ & Wasano K et al (28) \\
\hline p.E1841K & Substitution & Sweden & $\mathrm{AD}$ & Zetterberg E et al (29) \\
\hline p.V1560G & Substitution & Sweden & $\mathrm{AD}$ & Zetterberg E et al (29) \\
\hline p.R1165C & Substitution & Japanese & $\mathrm{AD}$ & Okano S et al (30) \\
\hline p.V34G & Substitution & Italian & $\mathrm{AD}$ & De Rocco D et al (31) \\
\hline p.R702S & Substitution & Italian & $\mathrm{AD}$ & De Rocco D et al (31) \\
\hline p.M847_E853dup & Duplication & Italian & $\mathrm{AD}$ & De Rocco D et al (31) \\
\hline p.K1048_E1054del & Deletion & Italian & $\mathrm{AD}$ & De Rocco D et al (31) \\
\hline p.D1447Y & Substitution & Italian & $\mathrm{AD}$ & De Rocco D et al (31) \\
\hline p.R1162T & Substitution & Italian & $\mathrm{AD}$ & Vettore S et al (32) \\
\hline p.V1516M & Substitution & Italian & $\mathrm{AD}$ & Pecci A et al (33) \\
\hline p.R1557L & Substitution & Italian & $\mathrm{AD}$ & Pecci A et al (33) \\
\hline
\end{tabular}

$\mathrm{AD}$, autosomal dominant.

Table IV. Mutations in $\beta$-crystallin A4 associated with congenital cataracts.

\begin{tabular}{|c|c|c|c|c|}
\hline Amino acid changes & Mutation type & Family origin & Pattern of inheritance & (Refs.) \\
\hline p.L69P & Substitution & India & $\mathrm{AD}$ & Vanita V et al (37) \\
\hline p.F94S & Substitution & India & $\mathrm{AD}$ & Vanita V et al (37) \\
\hline p.G2D & Substitution & China & $\mathrm{AD}$ & Kumar M et al (38) \\
\hline p.D3Y & Substitution & Honduras & $\mathrm{AD}$ & Kumar M et al (38) \\
\hline p.L11S & Substitution & Denmark & $\mathrm{AD}$ & Kumar M et al (38) \\
\hline p.T19M & Substitution & India & $\mathrm{AD}$ & Kumar M et al (38) \\
\hline p.V28M & Substitution & India & $\mathrm{AD}$ & Kumar M et al (38) \\
\hline p.F32L & Substitution & China & $\mathrm{AD}$ & Kumar M et al (38) \\
\hline p.R33L & Substitution & India & $\mathrm{AD}$ & Kumar M et al (38) \\
\hline p.V44M & Substitution & China & $\mathrm{AD}$ & Kumar M et al (38) \\
\hline p.V44M & Substitution & USA & $\mathrm{AD}$ & Kumar M et al (38) \\
\hline p.W45S & Substitution & China & $\mathrm{AD}$ & Kumar M et al (38) \\
\hline p.D47N & Substitution & China & $\mathrm{AD}$ & Kumar M et al (38) \\
\hline p.P59L & Substitution & USA & $\mathrm{AD}$ & Kumar M et al (38) \\
\hline p.A9V & Substitution & China & $\mathrm{AD}$ & Zhai Y et al (18) \\
\hline p.G147V & Substitution & Pakistan & $\mathrm{AD}$ & Chen J et al (39) \\
\hline
\end{tabular}

AD, autosomal dominant.
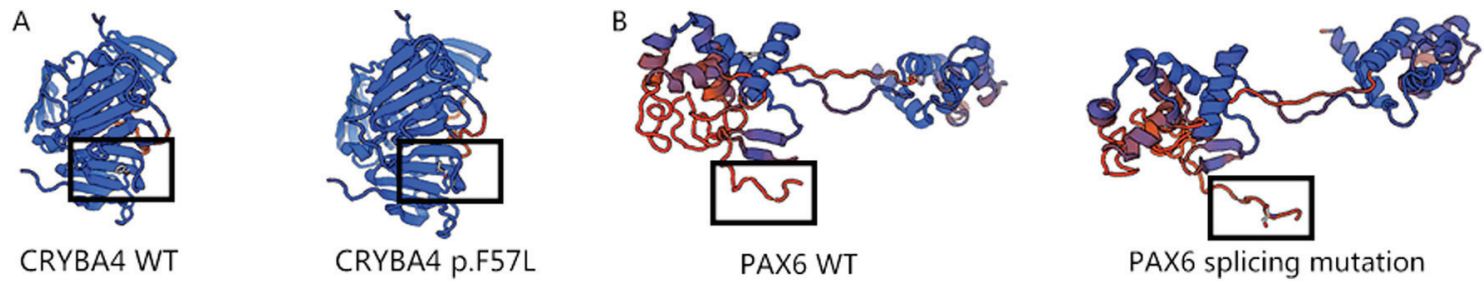

Figure 5. Three-dimensional models of WT and mutated CRYBA4 and PAX6 proteins. (A) CRYBA4 WT and CRYBA4 p.F57L mutation. (B) PAX6 WT and PAX6 splicing mutation. The locations of the mutations are indicated by rectangles. CRYBA4, $\beta$-crystallin A4; PAX6, paired box 6 ; WT, wild-type. 
genes analyzed, targeted exome sequencing to identify the mutations of the probands and Sanger sequencing for the remaining patients are highly efficient and economical (41). Of note, future studies will be performed in co-operation with the Reproductive Medicine Center of Peking University Third Hospital (Beijing, China) to develop potential prenatal diagnostic strategies to inhibit the effects of pathogenic mutations.

In addition, more information should be obtained to verify whether the cataracts caused by these mutations are progressive; however, it may not be possible to investigate certain features of the phenotypes of the affected individuals in the families in the future, as patients may undergo surgery to eliminate cataracts.

\section{Acknowledgements}

Not applicable.

\section{Funding}

The present study was funded by the Capital's Funds for Health Improvement and Research (grant no. 2018-2-4093).

\section{Availability of data and materials}

The datasets used and/or analyzed during the present study are available from the corresponding author on reasonable request.

\section{Authors' contributions}

$\mathrm{ZW}$ and $\mathrm{CH}$ analyzed the patient data and were major contributors in writing the manuscript. YS, HL, MZ and XL performed the ophthalmic examination of the patients. All authors read and approved the final manuscript.

\section{Ethics approval and consent to participate}

All participating individuals provided informed consent according to the tenets of the Declaration of Helsinki. The Medical Ethics Committee of Peking University Third Hospital (Beijing, China) approved all procedures of the present study.

\section{Patient consent for publication}

All participating individuals provided informed consent for publication.

\section{Competing interests}

The authors declare that they have no competing interests.

\section{References}

1. Hejtmancik JF: Congenital cataracts and their molecular genetics. Semin Cell Dev Biol 19: 134-149, 2008.

2. Robinson GC, Jan JE and Kinnis C: Congenital ocular blindness in children, 1945 to 1984. Am J Dis Child 141: 1321-1324, 1987.

3. Shiels A and Hejtmancik JF: Genetic origins of cataract. Arch Ophthalmol 125: 165-173, 2007.

4. Huang B and $\mathrm{He} \mathrm{W}$ : Molecular characteristics of inherited congenital cataracts. Eur J Med Genet 53: 347-357, 2010.
5. Francis PJ, Berry V, Bhattacharya SS and Moore AT: The genetics of childhood cataract. J Med Genet 37: 481-488, 2000.

6. Apple DJ, Ram J, Foster A and Peng Q: Elimination of cataract blindness: A global perspective entering the new millenium. Surv Ophthalmol 45 (Suppl 1): S1-S196, 2000.

7. Santana A and Waiswo M: The genetic and molecular basis of congenital cataract. Arq Bras Oftalmol 74: 136-142, 2011.

8. Gill D, Klose R, Munier FL, McFadden M, Priston M, Billingsley G, Ducrey N, Schorderet DF and Héon E: Genetic heterogeneity of the Coppock-like cataract: A mutation in CRYBB2 on chromosome 22q11.2. Invest Ophthalmol Vis Sci 41: 159-165, 2000

9. Liu Q, Wang KJ and Zhu SQ: A novel p.G112E mutation in BFSP2 associated with autosomal dominant pulverulent cataract with sutural opacities. Curr Eye Res 39: 1013-1019, 2014.

10. Kunishima S, Kojima T, Matsushita T, Tanaka T, Tsurusawa M, Furukawa Y, Nakamura Y, Okamura T, Amemiya N, Nakayama T, et al: Mutations in the NMMHC-A gene cause autosomal dominant macrothrombocytopenia with leukocyte inclusions (May-Hegglin anomaly/Sebastian syndrome). Blood 97: 1147-1149, 2001.

11. Choi Y and Chan AP: PROVEAN web server: A tool to predict the functional effect of amino acid substitutions and indels. Bioinformatics 31: 2745-2747, 2015.

12. Sim NL, Kumar P, Hu J, Henikoff S, Schneider G and Ng PC: SIFT web server: Predicting effects of amino acid substitutions on proteins. Nucleic Acids Res 40: W452-W457, 2012.

13. Schwarz JM, Rödelsperger C, Schuelke M and Seelow D MutationTaster evaluates disease-causing potential of sequence alterations. Nat Methods 7: 575-576, 2010.

14. Adzhubei IA, Schmidt S, Peshkin L, Ramensky VE, Gerasimova A, Bork P, Kondrashov AS and Sunyaev SR: A method and server for predicting damaging missense mutations. Nat Methods 7: 248-249, 2010

15. Waterhouse A, Bertoni M, Bienert S, Studer G, Tauriello G, Gumienny R, Heer FT, de Beer TAP, Rempfer C, Bordoli L, et al: SWISS-MODEL: Homology modelling of protein structures and complexes. Nucleic Acids Res 46: W296-W303, 2018.

16. Choi BY, Park G, Gim J, Kim AR, Kim BJ, Kim HS, Park JH, Park T, Oh SH, Han KH and Park WY: Diagnostic application of targeted resequencing for familial nonsyndromic hearing loss. PLoS One 8: e68692, 2013.

17. Sun W, Xiao X, Li S, Guo X and Zhang Q: Exome sequencing of 18 Chinese families with congenital cataracts: A new sight of the NHS gene. PLoS One 9: e100455, 2014.

18. Zhai Y, Li J, Yu W, Zhu S, Yu Y, Wu M, Sun G, Gong X and Yao K: Targeted exome sequencing of congenital cataracts related genes: Broadening the mutation spectrum and genotype-phenotype correlations in 27 Chinese Han families. Sci Rep 7: 1219, 2017.

19. Bu J, He S, Wang L, Li J, Liu J and Zhang X: A novel splice donor site mutation in EPHA2 caused congenital cataract in a Chinese family. Indian J Ophthalmol 64: 364-368, 2016.

20. Churchill AJ, Hanson IM and Markham AF: Prenatal diagnosis of aniridia. Ophthalmology 107: 1153-1156, 2000.

21. Shiels A and Hejtmancik JF: Mutations and mechanisms in congenital and age-related cataracts. Exp Eye Res 156: 95-102, 2017.

22. Graw J: Genetics of crystallins: Cataract and beyond. Exp Eye Res 88: 173-189, 2009.

23. Aoki T, Kunishima S, Yamashita Y, Minamitani K and Ota S: Macrothrombocytopenia with congenital bilateral cataracts: A phenotype of MYH9 disorder with Exon 24 indel mutations. J Pediatr Hematol Oncol 40: 76-78, 2018.

24. Seri M, Pecci A, Di Bari F, Cusano R, Savino M, Panza E, Nigro A, Noris P, Gangarossa S, Rocca B, et al: MYH9-related disease: May-Hegglin anomaly, Sebastian syndrome, Fechtner syndrome, and Epstein syndrome are not distinct entities but represent a variable expression of a single illness. Medicine (Baltimore) 82: 203-215, 2003.

25. Miyazaki K, Kunishima S, Fujii W and Higashihara M: Identification of three in-frame deletion mutations in MYH9 disorders suggesting an important hot spot for small rearrangements in MYH9 exon 24. Eur J Haematol 83: 230-234, 2009.

26. Saposnik B, Binard S, Fenneteau O, Nurden A, Nurden P, Hurtaud-Roux MF and Schlegel N; FrenchMYH9 networka: Mutation spectrum and genotype-phenotype correlations in a large French cohort of MYH9-Related Disorders. Mol Genet Genomic Med 2: 297-312, 2014. 
27. De Rocco D, Pujol-Moix N, Pecci A, Faletra F, Bozzi V, Balduini CL and Savoia A: Identification of the first duplication in MYH9-related disease: A hot spot for unequal crossing-over within exon 24 of the MYH9 gene. Eur J Med Genet 52: 191-194, 2009.

28. Wasano K, Matsunaga T, Ogawa K and Kunishima S: Late onset and high-frequency dominant hearing loss in a family with MYH9 disorder. Eur Arch Otorhinolaryngol 273: 3547-3552, 2016.

29. Zetterberg E, Carlsson Alle MS, Najm J and Greinacher A: Thrombin generation in two families with MYH9-related platelet disorder. Platelets 27: 264-267, 2016.

30. Okano S, Takase M, Iseki K, Toriumi N, Kaneda M and Kunishima S: Genotype-phenotype correlation of the p.R1165C mutation in the MYH9 disorder: Report of a Japanese pedigree. J Pediatr Hematol Oncol 37: e352-e355, 2015.

31. De Rocco D, Zieger B, Platokouki H, Heller PG, Pastore A, Bottega R, Noris P, Barozzi S, Glembotsky AC, Pergantou H, et al: MYH9-related disease: Five novel mutations expanding the spectrum of causative mutations and confirming genotype/phenotype correlations. Eur J Med Genet 56: 7-12, 2013.

32. Vettore S, De Rocco D, Gerber B, Scandellari R, Bianco AM, Balduini CL, Pecci A, Fabris F and Savoia A: A G to C transversion at the last nucleotide of exon 25 of the MYH9 gene results in a missense mutation rather than in a splicing defect. Eur J Med Genet 53: 256-260, 2010.

33. Pecci A, Panza E, De Rocco D, Pujol-Moix N, Girotto G Podda L, Paparo C, Bozzi V, Pastore A, Balduini CL, et al: MYH9 related disease: Four novel mutations of the tail domain of myosin-9 correlating with a mild clinical phenotype. Eur J Haematol 84: 291-297, 2010.
34. Pecci A, Klersy C, Gresele P, Lee KJ, De Rocco D, Bozzi V, Russo G, Heller PG, Loffredo G, Ballmaier M, et al: MYH9-related disease: A novel prognostic model to predict the clinical evolution of the disease based on genotype-phenotype correlations. Hum Mutat 35: 236-247, 2014.

35. Pecci A, Panza E, Pujol-Moix N, Klersy C, Di Bari F, Bozzi V, Gresele P, Lethagen S, Fabris F, Dufour C, et al: Position of nonmuscle myosin heavy chain IIA (NMMHC-IIA) mutations predicts the natural history of MYH9-related disease. Hum Mutat 29: 409-417, 2008.

36. Billingsley G, Santhiya ST, Paterson AD, Ogata K, Wodak S, Hosseini SM, Manisastry SM, Vijayalakshmi P, Gopinath PM, Graw J and Héon E: CRYBA4, a novel human cataract gene, is also involved in microphthalmia. Am J Hum Genet 79: 702-709, 2006.

37. Vanita V, Guo G, Singh D, Ott CE and Robinson PN: Differential effect of cataract-associated mutations in MAF on transactivation of MAF target genes. Mol Cell Biochem 396: 137-145, 2014.

38. Kumar M, Agarwal T, Kaur P, Kumar M, Khokhar S and Dada R: Molecular and structural analysis of genetic variations in congenital cataract. Mol Vis 19: 2436-2450, 2013.

39. Chen J, Wang Q, Cabrera PE, Zhong Z, Sun W, Jiao X, Chen Y, Govindarajan G, Naeem MA, Khan SN, et al: Molecular genetic analysis of Pakistani families with autosomal recessive congenital cataracts by homozygosity screening. Invest Ophthalmol Vis Sci 58: 2207-2217, 2017.

40. Zhou G, Zhou N, Hu S, Zhao L, Zhang C and Qi Y: A missense mutation in CRYBA4 associated with congenital cataract and microcornea. Mol Vis 16: 1019-1024, 2010.

41. Chen K, Zhou YX, Li K, Qi LX, Zhang QF, Wang MC and Xiao JH: A novel three-round multiplex PCR for SNP genotyping with next generation sequencing. Anal Bioanal Chem 408: 4371-4377, 2016. 